\title{
LAS BASES HISTÓRICAS DE LA EVALUACIÓN DE PROGRAMAS Y PROYECTOS SOCIALES
}

\author{
Fernando Salamanca Osorio*
}

No es novedoso afirmar que la planificación social tiene evidentes bases históricas, especialmente en el ámbito de los movimientos sociales y en general acciones reivindicativas que han marcado la historia social del este siglo.

Menos frecuente es la reflexión sobre las bases históricas de las herramientas operacionales de la planificación social, y en particular la evaluación de programas y proyectos sociales.

La evaluación de programas es un componente de la planeación que ha experimentado un proceso de institucionalización, que ya lo hace formar parte de la operatoria normal del Estado, siendo una exigencia básica para la optimización de la inversión social (MIDEPLAN, 1992). A pesar de su carácter de herramienta técnica, existen significativas condicionantes sociales e históricas que delimitan su origen y aplicación. El conocimiento y análisis de tales determinantes son esenciales para los profesionales de las ciencias sociales que trabajan en el área de los programas y proyectos sociales.

El desarrollo de la evaluación de programas se puede graficar en dos secuencias paralelas, que solo recientemente experimentan una ligazón más estrecha; es conveniente entonces diferenciar el desarrollo de la evaluación ex ante de proyectos de la evaluación de resultados o impacto.

\section{LA EVALUACIÓN EX ANTE DE PROYECTOS}

La biografía intelectual de la evaluación ex ante de proyectos proviene del campo de las obras públicas y de la ingeniería de proyectos (Prest, 1965). Sus atisbos de formalización se sitúan a comienzos de siglo, con la progresiva necesidad del capitalismo de Estado de aumentar la magnitud de sus gastos, y por lo tanto justificar el incremento de la carga tributaria. Para limitar las presiones políticas de los contribuyentes, el Estado (especialmente en EE.UU.), desarrolla una metodología para evaluar la rentabilidad de las inversiones públicas, incluso con la expectativa de recuperar parte de la plusvalía territorial generada por tales inversiones en los propietarios de tierras o medios de producción (Prest, op. cit.).

El New Deal en EE.UU., naturalmente incremento y diversificó las inversiones públicas, con lo que el instrumental evaluativo adopto una mayor sofisticación, aunque siguió siendo aplicado a la rentabilidad de las obras públicas.

Sólo después de la II Guerra Mundial, la evaluación de proyectos experimenta un avance substancial, al incorporársele el bagaje teórico de la economía del bienestar, de corte neoclásico. Esta formalización conceptual le abrió la posibilidad de ampliar su aplicación a prácticamente todas las áreas en que el Estado orientaba sus inversiones.

*Académico Departamento de Sociología, Facultad de Ciencias Sociales, Universidad de Chile. 
De manera especial, se concentró la aplicación de conceptos de la economía del bienestar en el diseño de técnicas evaluativas costo-beneficio, y orientando su aplicación en los sectores sociales. Un punto central en esta reorientación se deriva de la evidencia empírica que las inversiones en capital humano (vía educación, salud y nutrición principalmente) tienen un definido impacto en el incremento de la productividad de la mano de obra. (Routlinger y Selowsky, 1975)

La mecánica de operación de la técnica costo-beneficio se popularizó con rapidez en los ámbitos de los planificadores y tomadores de decisiones. Se produce una rápida institucionalización, extendiéndose su aplicación a los países de Europa. Resulta notable el uso de la técnica costo-beneficio en los países europeos, donde el sistema político favorecía la expansión del gasto público. Tal es el caso de Inglaterra, durante la administración laborista (Self, 1975).

La evaluación costo-beneficio es incorporada a manuales de proyectos de Naciones Unidas y agencias de la cooperación internacional, y utilizada como instrumento de asignación de inversiones por organismos multilaterales como el Banco Mundial y el Banco Interamericano de Desarrollo (Payer, 1982).

La misma proliferación de la evaluación costo-beneficio, evidenció sus limitaciones, producto del irrealismo de los supuestos de la economía del bienestar, de hecho inexistentes, incluso en las más avanzadas economías de mercado. Los propugnadores de esta técnica se bifurcaron en dos modalidades de corrección de su formulación original. Una, que se podría calificar como la corriente más ortodoxa, que intenta generalizar los supuestos teóricos de la economía del bienestar, basados en la competencia perfecta al conjunto de la sociedad. En la práctica, la pretensión de estos evaluadores es colocar a la estructura económica en consonancia con los dictados de comportamiento económico supuestos por la técnica. Se advierte que esta opción es la asociada a los experimentos neoliberales como estilo de desarrollo; se trata de redefinir las características del capitalismo de Estado a los prerrequisitos de la economía neoclásica.

La otra vertiente derivó en una posición más pragmática, en relación a flexibilizar la técnica, ajustándola a las peculiaridades de la estructura capitalista real, en especial la que prima en países del Tercer Mundo. Se agregan además, en el cálculo de costos y beneficios, factores de equidad social y redistribución, especialmente en programas que involucren beneficiarios de menores ingresos (Silva, 1983).

La evolución anteriormente esbozada, permite visualizar una aproximación metodológica centrada en el cálculo económico, o más precisamente de índole contable y financiera. Se advierte su asociación con el desarrollo del capitalismo de Estado. La mayor funcionalidad de la técnica reside en la correcta asignación de recursos estatales en la formación de capital fijo e infraestructura. El mismo desarrollo capitalista la reorienta después a la racionalización de la acción estatal en la reproducción de la fuerza de trabajo (salud, educación, vivienda, etc.), aunque de hecho mantenga componentes de la evaluación de la infraestructura social, especialmente en la variante de evaluación costo-efectividad.

El período expansivo del gasto social en las economías de mercado, desde hace algún tiempo se ha estabilizado, y consecuentemente ha sido acompañado por la búsqueda de metodologías evaluativas lo más precisas posibles, para optimizar los ya críticos márgenes del gasto público social. La técnica costo-beneficio aparentemente cubrió durante un tiempo esta necesidad, pero su propio formalismo y axiomática ahistórica le impide ser un instrumento eficaz y válido de asignación de inversiones sociales. La superación de la 
crisis se intenta por las vías ya mencionadas, ya sea por la hipertrofia ortodoxa y neoliberal, o una adecuación pragmática a las economías de mercado propias de los países pobres. Sobre el éxito de algunas de estas aproximaciones sería aún prematuro emitir un juicio.

\section{EL DESARROLLO DE LA EVALUACIÓN DE RESULTADOS O IMPACTO}

La otra vertiente en la evaluación de programas se asocia a una dimensión psicosocial, y se la conoce usualmente como evaluación de resultados. También su génesis, al igual que la evaluación costo-beneficio, hay que rastrearla en el periodo posterior a la I Guerra Mundial, con las exigencias de mayor precisión de los programas sociales. En este caso, es importante la creciente importancia de la Psicología social aplicada, mediante el desarrollo de diseños experimentales y cuasiexperimentales en la modificación de la conducta.

La evaluación de resultados también se vio reforzada por la constatación empírica de la acción grupal en la conducta individual, especialmente en lo referente al cambio de actitudes.

Posteriormente a la II Guerra Mundial en EE.UU.,' proliferan los programas de desarrollo urbano, capacitación ocupacional y atención preventiva en salud, áreas aptas para el desarrollo de la Psicología y Sociología aplicada, y que permitieron un nuevo impulso a la evaluación de resultados en los programas sociales (Rossi, 1989).

Más tarde, se generalizan los programas de evaluación en gran escala, en la prevención de la delincuencia, rehabilitación penal y tratamientos psicoterapéuticos. Se advierte que la evaluación de resultados se concentra en proyectos muy específicos, al interior de sectores sociales; tales proyectos se implementan en contextos cerrados y con grupos de manipulación experimental más sencilla. De hecho, algunos sociólogos presentan la evaluación como una forma concreta de investigación social, con todas las fases de diseño investigativo implicado en ello. En este período — aproximadamente la década del 60 - se caracteriza por la institucionalización científica y profesional de la evaluación de resultados. Debe destacarse que es un período en EE.UU., y secundariamente en Europa, marcado por el incremento de los movimientos sociales que demandan mayor cobertura y diversificación de los programas antipobreza. La respuesta estatal, expresada en múltiples proyectos sirvió como campo de aplicación de la evaluación de resultados.

La discutible eficacia de los programas erradicatorios de la pobreza en los países desarrollados, ha redundado que la evaluación de resultados derive en una postura más orientada a las actividades políticas y administrativas, en otros términos, es una actividad científica que colabora en la toma racional de decisiones. De este modo, se valora el fin político de la evaluación. Hay una transición de una perspectiva cientificista a una política; obviamente esto influye en una pérdida de rigidez de los cánones metodológicos de la evaluación, en función de su adaptación al tiempo y a la lógica de la negociación política (Wolf, 1975) 


\section{LA SITUACIÓN ACTUAL EN LA EVALUACIÓN}

En síntesis, tanto la evaluación costo-beneficio como la de resultados, a pesar de sus desarrollos paralelos, provienen de un mismo contexto intelectual, que en última instancia se localiza en las corrientes empiricistas y utilitarias de Inglaterra del siglo XVII y XVIII (Stone, 1978). Es explicable luego que en estas técnicas su confianza en la medición y objetivación del comportamiento externo, la creencia en la manipulación y perfeccionamiento de la conducta humana, en especial al logro del máximo bienestar individual. Más tarde, una de ellas (costo-beneficio) se asociaría con la versión económica del utilitarismo inglés; y la otra (de resultados) con el empirismo conductual.

Actualmente, el esfuerzo de aunar ambas perspectivas obedece a la necesidad de articular un instrumento estandarizado para la amplia variedad de programas sociales, en especial en un marco de optimización y focalización de programas sociales en sectores de extrema pobreza.

La evaluación ha asumido el rango de una herramienta de decisión política, aunque de su utilización en este plano caben lecturas alternativas, que se pueden esbozar en cuatro estilos:

a) La evaluación utilizable como relaciones públicas, en tanto estas técnicas se asumen como forma de publicitar resultados favorables para una administración o institución. Tal promoción puede darse en medios de difusión de masas, o en forma más sutil, vía el impacto en públicos especializados como planificadores sociales o la comunidad científica.

A su vez, tales relaciones públicas pueden tener como interlocutor auditorios estratégicos como entes financistas (oficinas de presupuestos, fundaciones, agencias de la cooperación internacional) oficinas sectoriales o centrales de planeación, grupos de presión profesionales, usuarios de los programas. Según las circunstancias, estos distintos referentes resultan apoyos cruciales para la mantención o expansión de un programa.

Debe destacarse el rol inhibidor o crítico de la comunidad científica para el evaluador en tanto relacionador público, tendiendo más bien una creciente formalización del status del evaluador y su inserción en sociedades científicas o publicaciones especializadas.

b) La actitud cínica, que sería una deformación de la actitud orientada a las relaciones públicas. En este caso, la credibilidad interna de la eficacia de la evaluación es mínima; se la considera un ejercicio artificioso de marcos conceptuales o estadísticos, que ilustran con ribetes científicos conclusiones de sentido común sobre la bondad de programas sociales.

A su vez, se reconoce que la sociedad considera a la evaluación como un recurso prestigioso para la toma de decisiones. La actitud cínica - aunque no se explicita en forma tan cruda - ha adquirido cierta importancia en el último tiempo en los países más desarrollados, por el fracaso relativo de las estrategias evaluativas en las décadas pasadas, y por la consiguiente anarquía de las técnicas en uso en la actualidad.

Se considera que los planificadores gratifican - social como económicamente- el ejercicio evaluativo, a conciencia de las limitaciones de las técnicas utilizadas, en consideración de la burocratización de los servicios estatales sociales, y los intereses creados asociados a tal tecnoestructura. Mientras esté vigente esta situación, el evaluador mantendrá su actividad, aunque sin la convicción personal de su utilidad o eficacia. 
c) La orientación racional, justamente lo contrario de las dos anteriores, postula una amplia confianza en las técnicas evaluativas y en su óptima utilización por los tomadores de decisiones. Su actitud racionalista se basa en la superioridad del discurso tecnocrático y su aceptación universal. Eventualmente, cuando se constatan barreras precientíficas, éstas serían progresivamente superadas por las técnicas de programación social. A su vez supone que el aparato administrativo e institucional acoge positivamente los resultados entregados por la evaluación y se retroalimenta en función de ésta. También se facilita dentro del aparato estatal, la incorporación de las técnicas evaluativas en instituciones sociales, inicialmente refractarias a la aplicación de esta metodología.

De hecho, no se cree que existan diferencias substanciales en las estrategias evaluadoras entre los distintos sectores sociales. Toda conducta modificada por un programa social, cualquiera sea su índole, puede ser evaluada, y este criterio puede ser - o debería ser- asumido por toda entidad adscrita a la planificación social.

d) La visión de la evaluación como ayuda a la toma de decisiones, que sería una adaptación realista de la opción racional. Se acepta que hay diferencias de magnitud entre el discurso del científico, el del planificador y el del tomador de decisiones. Éstos responden a lógicas diferentes, no siendo posible establecer un rango de superioridad o jerarquía entre ellos.

Se considera que la racionalidad que prima en la esfera de la planificación social es limitada, y sujeta a restricciones de tiempo y sentido de oportunidad de la decisión. A su vez, la definición del rol del evaluador está dada por su inserción en la tecnoestructura, lo que introduce un mayor sentido de realismo y pragmatismo frente a la operatoria del sistema político.

\section{LA SITUACIÓN DE LA EVALUACIÓN EN CHILE}

En nuestro país se constata que la formulación definida de programas sociales tiene una larga trayectoria, originándose a comienzos de siglo, e institucionalizándose posteriormente en programas sectorializados que no diferían mayormente de la oferta de servicios en otros países del mundo; sin embargo la incorporación de la evaluación como elemento importante dentro de la formulación y desarrollo de programas sociales es de reciente data.

En el marco de la modernización del Estado, adquiere especial relevancia la inserción de la inversión social dentro del ciclo de inversión pública, dentro del cual se establece como norma la evaluación ex ante de proyectos, además de otros mecanismos de incentivo a la eficiencia en la gestión de políticas sociales. Sin embargo, las raíces de la evaluación provienen de la aplicación dentro de prácticamente todas las esferas gubernamentales, de los postulados de la economía neoliberal. Se ha insistido que la evaluación costo-beneficio es una derivación de la economía neoclásica. Los programas sociales, luego, deberían legitimarse como actividades públicas, sólo a través del discurso formal de la economía del bienestar.

Inicialmente, esta redefinición de la justificación de la programación social, se limitó a un debate intelectual iniciado por M. Friedman, y en Chile, sobre todo desde 1975 por A. Harberger en círculos académicos y políticos proclives al enfoque neoliberal. 
Sólo a fines de la década del 70, con la hegemonía política efectiva del discurso neoliberal en la asignación de recursos, comienzan los primeros atisbos de aplicación de evaluación de proyectos a sectores sociales. No obstante, sería la crisis económica que se generaliza a partir de 1982, que permite un uso efectivo de la evaluacion, vía técnica costo-beneficio. En este período la expansión del gasto social llega a su límite, encontrándose éste en alrededor de la mitad del gasto público.

La misma profundidad de la crisis, haría prácticamente imposible expandir aún más el gasto público, salvo asumiendo decisiones de política lesivas a la satisfacción mínima de necesidades básicas.

Se impone entonces una preocupación por la eficacia y sobre todo por la eficiencia de los programas sociales, enfatizando en primer término, la focalización los programas sociales, limitando al máximo la oferta universal de subsidios, reorientándolos como redes sociales circunscritas a los sectores en extrema pobreza, incluso estableciendo subestratos al interior de ésta.

De este modo la evaluación costo-beneficio (en especial la de tipo ex ante) adquiere una importancia central, representando una de las varias formas de legitimación de la tecnocracia gubernamental, y siendo además uno de los mejores ejemplos de la eficacia operativa de la economía de bienestar.

La racionalización del gasto público social orienta las estrategias evaluativas a los programas sociales que incorporan mayor volúmenes de recursos (educación, salud, alimentación, etc.). Naturalmente, esta orientación significó además un cambio radical en la formulación de los programas en estos sectores, lo que trajo aparejada una deslegitimación en la burocracia tradicional de la evaluación costo-beneficio y costo-efectividad, sin una alternativa de igual rigor conceptual, agravando la situación el contexto autoritario en que se implementan estas modalidades evaluativas.

Debe destacarse que la burocracia estatizante de fines de la década del 70 , carecía de la ideología de centro o izquierda que tradicionalmente en Chile respaldaba la acción del Estado, de allí su debilidad estructural para definir un proyecto estatal propio, aparte del de la economía neoclásica. El desplazamiento de estos atomizados grupos fue prácticamente inevitable. Esta marginación, sutilmente evidente en ODEPLAN, Ministerio de Educación, Salud, etc., no estuvo exenta de dificultades, y en ciertos casos la negociación interna distorcionó la finalidad original de la propuesta neoliberal, generando desde el enfoque de la economía del bienestar híbridos programáticos que hasta la fecha subsisten.

El copamiento interno del Estado, por parte de la tecnocracia neoliberal, implicó el uso de la evaluación como instrumento político, criticando la eficacia —en relación a cánones técnicos - de las políticas tradicionales, presionando por la modificación drástica de éstas.

El marco interpretativo de esta presión por la focalización y evaluación ex ante y cuantitativa de programas sociales es ejemplificado por el proceso de modernizaciones a partir de 1980. La crisis recesiva posterior acelera este proceso, siendo indudable que persiste hasta nuestros días, como lo ilustra la diversas metodologías de formulación y evaluación de proyectos vigentes en el Estado. Sin embargo, dentro del propio aparato de gobierno la legitimidad técnica de estas estrategias evaluativas despierta críticas por su limitada capacidad para discriminar la opción neoliberal. 


\section{EVALUACIÓN Y ANTAGONISMO SOCIAL}

El proceso evaluativo de programas en el modelo neoliberal ha tenido escaso impacto público, marginando otros actores que no sean los propios impulsores técnicos de la evaluación. El concepto de sociedad evaluativa es aún muy lejano.

Un caso aislado, pero interesante, son los procesos sociales y debates técnicos suscitados por el intento de modificación del Programa Nacional de Alimentación Complementaria (PNAC) introduciendo variaciones en la oferta de leche por sopa, puré u otros tipos de complemento alimentario. Tal modificación del tipo de entrega de una institución del Estado era ya objeto de investigación empírica desde 1975 por diferentes organismos tanto extranjeros como nacionales. En este sentido, la evaluación del PNAC siguió en el plano investigativo, rematando en evaluaciones costo-beneficio, que sugerian la modificación de los programas usuales a la fecha (Torche, 1980). En este proceso se pueden esbozar los siguientes pasos:

- evaluación del impacto e la ingesta de leche en la variación positiva de una variable de calidad de vida como la mortalidad infantil;

- una vez comprobada estadísticamente la magnitud de tal impacto, se determina la relación costo-eficiencia de las distintas formas de intervención nutricional;

- finalmente se determina la tasa de retorno por grupos de edad, para establecer el rango de edad en que la intervención es más eficaz.

De este modo, sucesivas evaluaciones permitieron establecer la posibilidad técnica de optimizar el gasto en este programa, focalizándolo en los grupos de mayor riesgo, y reemplazando el tipo de intervención en los grupos objetivos que serían marginados del nuevo diseño de programa. El paso siguiente en la tecnoestructura era la modificación real del programa.

Lo sucedido posteriormente es relativamente conocido, se expresó en la reacción masiva de rechazo de los sectores afectados; también se evidenció la repulsa en la comunidad científica y profesional (Serra, 1984; Scherman,1988). Tal reacción define una debilidad substancial de la evaluación costo-eficiencia y costo-beneficio en el actual esquema, cual es la marginación de los múltiples actores relacionados con este proceso, aparte del planificador y evaluador social.

La reacción popular se concentró, más que en una impugnación metodológica de la técnica en uso, en la indignación por la pérdida de un beneficio del Estado, de cobertura generalizada en sectores pobres. Efectivamente, los estudios de cobertura de programas subsidiarios estatales muestran que en el PNAC el acceso al programa es casi total, no así en otros programas erradicatorios. La hipersensibilidad popular era correlativa al deterioro generalizado de sus necesidades básicas en el actual período.

La movilización, tuvo como detonante la implementación autoritaria de una evaluación tecnocrática aislada del contexto político social nacional, que ya se encontraba en un período de radicalización, especialmente en el marco poblacional, que es donde se localizan los lugares de otorgamiento de servicios (postas y policlínicos). La visibilidad de la instancia que limitaba sus servicios sociales facilitó la expresión de rechazo.

De este modo, la alternativa programática careció de una correcta caracterización del contexto social que iba a ser impactado por la evaluación.

Aparte del caso mencionado del PNAC, se han realizado otras evaluaciones dentro del aparato del Estado, que han afectado programas de menor cobertura, en estos casos los 
usuarios han sido receptores pasivos de la modificación programatica. Tal es el caso de las modificaciones a las modalidades de atención preescolar, con la creación de los Centros de aprendizaje y desarrollo del lenguaje (CADEL, posteriormente Jardines Familiares). Los beneficiarios no han sido coparticipes del proceso evaluativo desde su gestación interna, ni tampoco como referentes externos. Las características de los programas hacen difícil la concertación de actores, a lo que se agrega su gran dispersión territorial.

\section{LA OPCIÓN PARTICIPATIVA EN LA EVALUACIÓN}

El destino de la evaluación en Chile, hasta ahora esta regido aún por patrones teórico y metodológicos neoliberales. Aún más solo recientemente se esta utilizando la vertiente evaluativa de resultados, a pesar que comparte con la evaluación costo-beneficio una misma tradición empiricista.

Resultaría excesivamente simplista pretender que esta opción evaluativa es una mera deducción operacional de un estilo de desarrollo neoliberal. Ella se aplica con modificaciones significativas en países con economías mixtas y socialistas. Un componente central del discurso planificador es el uso de criterios científicos de selección y evaluación de proyectos, en tanto son exigencias de asignación, control y retroalimentación de todo programa o proyecto de inversión social, y en especial en países en desarrollo en situaciones de escasez del excedente social.

La insistencia en focalizar al máximo los beneficios del Estado es razonable, más aún cuando cumple la doble función de comprometer eficientemente los recursos del Estado y lograr el máximo bienestar individual y familiar en los sectores más deprivados de la población.

Las fallas del enfoque aplicado en Chile en el plano estatal, no se encuentran en las modalidades evaluativas predominantes en sectores alternativos a los programas oficiales. En estos casos prima la evaluación conocida como iluminativa, orientada a dimensiones profundas del comportamiento (ideología, creencias, etc.), utilizando instrumental metodológico de corte cualitativo que registran más válidamente los procesos cotidianos de transformación del sujeto (Richards, 1985).

El interlocutor válido de la evaluación, más que el tomador de decisiones es la propia comunidad receptora (y a menudo actora) del programa o proyecto.

La evaluación es de índole grupal y basada en criterios de autovaloración, siendo secundaria la proveniente del grupo técnico. Para los evaluadores de programas de instituciones alternativas, la base de su estilo evaluativo es una opción básicamente fenomenológica, ajena a la concepción empiricista propia de la evaluación de resultados con base a diseños cuasiexperimentales. La opción iluminativa obviamente es radicalmente distinta a la economía del bienestar que subyace en la técnica costo-beneficio.

Más allá de los indudables méritos de la evaluación iluminativa utilizada en instituciones alternativas, subyace la interrogante sobre la factibilidad de una estrategia de este tipo en el ciclo gubernamental de inversión pública, que utiliza primodialmente la evaluación ex ante ya sea costo-beneficio o costo-efectividad. Se plantean al Estado desafíos que obligan a la modificación de estructuras institucionales, por ejemplo, la existencia de políticas sectoriales nacionales de carácter homogéneo, con una estructura administrativa central. En este patrón, debería adaptarse una metodología evaluativa de 
tipo estandarizado en que primaria el registro de datos conductuales, principalmente relacionados a variables contextuales. La información de índole psicosocial sería de difícil obtención, sorteando la dificultad con escalas e índices de ponderación variable.

Al parecer, por las deficiencias de la conformación tradicional del Estado, la tendencia en América Latina es a incentivar una reforma radical en la forma de planificar, acentuando una gestión estatal descentralizada, disminuyendo los límites entre el Estado formal y la sociedad civil, al menos en los niveles locales de la acción pública; en estas circunstancias sería factible aplicar un criterio pragmático en evaluación de programas y proyectos. El pragmatismo debería orientarse a seleccionar la combinación óptima de técnicas, dadas las siguientes situaciones:

- la escala de los programas sociales, que van del rango nacional al intracomunal y vecinal;

- la heterogeneidad de los grupos objetivos, diferenciables por edad, sexo, región, sector, etnia, etcétera;

- la duración de los programas y la extensión de sus efectos en el tiempo;

- la mayor o menor incorporación de tecnología o conocimientos especializados;

- la existencia y calidad de la estructura administrativa para aplicar las estrategias evaluativas.

Estos criterios, y otros más no categorizados, obligan al diseño de un continuo de estrategias evaluativas que irían desde las evaluaciones de monitoreo continuo de programas con indicadores cuantitativos de insumos, proceso, resultado e impacto derivados de Censos o Encuestas de Hogares, hasta las de corte psicosocial y antropológico basadas en pequeñas muestras o estudios de casos. Unas deberían reforzar o complementar a las otras, evitando la aplicación de instrumentos únicos de evaluación.

La participación de la población en el proceso evaluativo esta determinada por la ingerencia directa del grupo de beneficiarios en el programa, tanto en la fase de formulación e implementación, como de evaluación. Otra vía de participación es de tipo representativo en las instancias locales, regionales y nacionales de toma de decisiones (Concejales municipales o consejeros regionales, Parlamento). Esta última adquiere cada vez mayor importancia por la delegación de atribuciones en la decisión de proyectos de inversión, que por las características del Estado en el período post recesivo son de hecho inversiones sociales

Resulta previsible la tendencia de las estrategias evaluativas a nivel estatal por técnicas de evaluación costo-beneficio por su relativa o engañosa sencillez, acompañada por el manejo de datos cuantitativos. Como también es razonable suponer que las estrategias de evaluación de programas gubernamentales menores o de organizaciones no gubernamentales se limiten a técnicas iluminativas, al margen de factores de costo o eficiencia de su impacto.

Para que una estrategia combinada, de eficiencia (costo-beneficio y costo-efectividad) como de impacto (cualitativas o iluminativas) tenga cierto grado viabilidad, es necesario que la administración del Estado este efectivamente descentralizada y desconcentrada, transfiriendo poder decisorio a los organismos sociales, en especial a nivel local. Es central que esta autonomía incluya una adaptabilidad de los criterios nacionales de formulación y evaluación de proyectos de inversión, sorteando la dificultad actual de aplicación nacional de criterios de evaluación (especialmente en la fase ex ante) por encima de las peculiaridades de las necesidades básicas en cada región y localidad. 


\section{REVISTA DE SOCIOLOGÍA}

También es necesario una socialización en la población de su rol vital como ente evaluador activo, al respecto se hace necesario una educación para la evaluación social, como una mayor incorporación del científico social en la programación participativa.

Es crucial romper la barrera entre el conocimiento técnico administrado por el evaluador, y los datos que pasivamente son extraídos del grupo de beneficiarios. La búsqueda de un lenguaje común, implica de cualquier modo la remoción de las múltiples barreras que actualmente alejan la programación y la evaluación social de la base social.

Por último resulta importante la experiencia de los fondos de inversión que mediante sistemas más flexibles de diseño, evaluación y supervisión de proyectos esta demostrando que es posible estructurar evaluaciones multicriterio sin perder de vista la óptima asignación de la inversión social (Salamanca, 1993). El sistema evaluativo de fondos de inversión, ilustrado en Chile con el Fondo de Solidaridad e Inversión Social (Fosis) aún limitado por imperfecciones derivadas de la confiabilidad y validez de sus instrumentos de evaluación, representa un avance en relación a un uso rígido de las tradicionales evaluaciones costo-impacto.

Las metodologías costo-impacto ex ante han demostrado una pobre capacidad predictiva para estimar la sustentabilidad a mediano y largo plazo de proyectos, tanto sociales como productivos. Una posible interpretación es su tendencia a sobreestimar retornos económicos de la inversión (Pohl, 1992), descuidando factores de experiencia organizacional, y en especial la nula consideración que hacen de los beneficiarios en las diferentes fases de la formulación, implementación y evaluación de proyectos (World Bank, 1992)

\section{BIBLIOGRAFÍA}

MIDESLAN, Inversión pública eficiencia y equidad. Departamento de inversiones. Santiago. 1992

PAYER, CHERYL, The World Bank: a critical analysis. Monthly Review Press. London. 1982

Pohl., G. and M. DLbravko, 1992, "Project evaluation and uncertainty in practice", The World Bank Ecomomic Review, Vol. 6.

PREST, A.R. y TURVEY, R. "Un estudio de costos y beneficios" en The Economic Journal, Dic. 1965. N'167, Vol. LXXV (2). $255-277$

Richards, Howard, La evaluación de la acción cultural. CIDE. Santiago. 1985.

Rossi. Peter and H. FreEma.v, 1989, Evaluation: a systematic approach. Sage. Beverly Hills.

ROUtLiNger, S. y SELOWSKy, M. Malnutrition and polierty: nagnitude and policy options, World Bank, Occasional Paper No 167, 1976, Washington

SALAmanca, F. Apuntes sobre formulación y evaluación de proyectos sociales. Departamento de Sociología. Universidad de Chile, Santiago, 1993.

SCHERMAN. JORGE, Las políticas de la salud y su impacto en los sectores populares: Chile 1974-1986. PET. Santiago. 1986

SELF, PETER, Econocrats and the policy process. MacMillan Press. 1975. Londres.

SERRA, IVÁ y LORETo CERDA, "Intento de evaluación del programa de alimentación complementaria" en Cuadernos médico-sociales. Vol. XXV, $\mathrm{N}^{\circ} 1$. Marzo 1984

Silva, IVÁs, Algunos conceptos básicos acerca de la evaluación financiera econóntica y social de proyectos. Documento PR-2. Programa de capacitación. ILPES. 1983

STONE, ERNEST, "Assumptions underlying evaluation models" en Educational research. March 1878.

WOLf, ROBERT "Trial by jury: a new evaluation method" en Phi Delta Kappan. Vol 57. No 3, November 1975.

World Bank, Informe anual 1992. World Bank. Washington, D.C. 1992. 